\title{
Lattice Theories with Nonlinearly Realized Chiral Symmetry
}

\author{
S. Chandrasekharan ${ }^{\mathrm{a}}$, M. Pepe ${ }^{\mathrm{b}}$, F. D. Steffen ${ }^{\mathrm{b} *}$ and U.-J. Wiese ${ }^{\mathrm{b} \dagger}$ \\ ${ }^{a}$ Department of Physics, Duke University, P.O. Box 90305, Durham NC 27708-0305, USA \\ ${ }^{\mathrm{b}}$ Institute for Theoretical Physics, Bern University, Sidlerstrasse 5, CH-3012 Bern, Switzerland
}

We present the lattice formulation of effective Lagrangians in which chiral symmetry is realized nonlinearly on the fermion fields. In this framework both the Wilson term removing unphysical doubler fermions and the fermion mass term do not break chiral symmetry. Our lattice formulation allows us to address non-perturbative questions in effective theories of baryons interacting with pions and in models involving constitutent quarks interacting with pions and gluons. With the presented methods, a system containing a non-zero density of static baryons interacting with pions can be studied on the lattice without encountering a complex action problem. This might lead to new insights into the phase diagram of strongly interacting matter at non-zero chemical potential.

\section{INTRODUCTION AND OVERVIEW}

In order to realize chiral symmetry on the lattice, it is by now understood that the lattice Dirac operator must satisfy the Ginsparg-Wilson relation and not anticommute with $\gamma_{5}$ as assumed by the Nielsen-Ninomiya theorem [1]. We present an alternative view at chiral symmetry on the lattice within low-energy effective theories (cf. [2] for details). Based on spontaneous chiral symmetry breaking, effective Lagrangians can be constructed which involve an explicit pion field in addition to phenomenological baryon [3] or constituent quark and gluon fields [4]. The pion field allows one to realize chiral symmetry nonlinearly on the fermion fields. The fermion mass term then does not break chiral symmetry [3]. We formulate such effective theories on the lattice. The nonlinear realization of chiral symmetry avoids the Nielsen-Ninomiya theorem and allows us to remove the doubler fermions with a Wilson term while maintaining exact chiral symmetry on the lattice. Our lattice formulation can be used to address non-perturbative questions in effective theories, to study static baryons at non-zero chemical potential, and to test the chiral quark model [4].

\footnotetext{
*Fellow within the Postdoc-Programme of the DAAD.

$\dagger$ On leave from MIT.

Work supported by the DOE under grant DOE-FG0296ER40945 and by the Schweizerischer Nationalfond.
}

\section{NONLINEAR REALIZATION OF CHIRAL SYMMETRY}

Let us illustrate the nonlinear realization of chiral symmetry for a low-energy effective theory of pions and nucleons introduced originally in [3].

\subsection{Continuum Formulation}

The chiral symmetry group of QCD in the limit of $N_{f}$ massless quark flavors $G=S U\left(N_{f}\right)_{L} \otimes$ $S U\left(N_{f}\right)_{R} \otimes U(1)_{B}$ is spontaneously broken to the vector subgroup $H=S U\left(N_{f}\right)_{L=R} \otimes U(1)_{B}$. Thus, massless Goldstone boson fields $U(x) \in$ $G / H=S U\left(N_{f}\right)$ govern the low-energy dynamics of QCD. The corresponding low-energy effective theory is given to lowest order in the Goldstone boson momenta by the following action $[5,6]$ :

$S[U]=\int d^{4} x \frac{F_{\pi}^{2}}{4} \operatorname{Tr}\left[\partial_{\mu} U^{\dagger} \partial_{\mu} U\right]$

where $F_{\pi}$ is the pion decay constant. By construction, this action is invariant under global chiral rotations, $L \otimes R \in S U\left(N_{f}\right)_{L} \otimes S U\left(N_{f}\right)_{R}$, under which the Goldstone boson field transforms as

$U(x) \rightarrow U^{\prime}(x)=L U(x) R^{\dagger}$.

To extend this theory to a low-energy effective theory for baryons, an auxiliary field is defined:

$u(x)=U(x)^{1 / 2}$, 
which transforms such that (2) is satisfied:

$u(x) \rightarrow u(x)^{\prime}=L u(x) V(x)^{\dagger}=V(x) u(x) R^{\dagger}$.

The matrix $V(x) \in S U\left(N_{f}\right)$ is a local realization of the global symmetry $G$,

$$
\begin{aligned}
V(x) & =R\left[R^{\dagger} L U(x)\right]^{1 / 2}\left[U(x)^{1 / 2}\right]^{\dagger} \\
& =L\left[L^{\dagger} R U(x)^{\dagger}\right]^{1 / 2} U(x)^{1 / 2} .
\end{aligned}
$$

Treating $V(x)$ basically as a flavor gauge transformation, chiral symmetry can be realized nonlinearly on the fermion fields.

Concentrating on the case $N_{f}=2$, a nucleon field - described by a Dirac spinor $\Psi(x)$ and $\bar{\Psi}(x)$ in the fundamental representation of $S U\left(N_{f}=\right.$ $2)$ - transforms under the nonlinear realization of chiral symmetry as follows

$\Psi(x) \quad \rightarrow \quad \Psi(x)^{\prime}=V(x) \Psi(x)$,

$\bar{\Psi}(x) \quad \rightarrow \quad \bar{\Psi}(x)^{\prime}=\bar{\Psi}(x) V(x)^{\dagger}$.

A "flavor covariant" derivative can then be constructed with the composite anti-Hermitean field

$v_{\mu}(x)=\frac{1}{2}\left[u(x)^{\dagger} \partial_{\mu} u(x)+u(x) \partial_{\mu} u(x)^{\dagger}\right]$

transforming as a vector "gauge" field

$v_{\mu}(x) \rightarrow v_{\mu}^{\prime}(x)=V(x)\left[v_{\mu}(x)+\partial_{\mu}\right] V(x)^{\dagger}$.

A composite Hermitean field can be build as well

$a_{\mu}(x)=\frac{i}{2}\left[u(x)^{\dagger} \partial_{\mu} u(x)-u(x) \partial_{\mu} u(x)^{\dagger}\right]$,

which transforms as an axial vector

$a_{\mu}(x) \rightarrow a_{\mu}^{\prime}(x)=V(x) a_{\mu}(x) V(x)^{\dagger}$.

Now we can write down the leading terms of the Euclidean action describing a chirally symmetric low-energy effective theory for nucleons and pions

$$
\begin{aligned}
S[U, \bar{\Psi}, \Psi]= & S[U]+\int d^{4} x\left\{\bar{\Psi} \gamma_{\mu}\left(\partial_{\mu}+v_{\mu}\right) \Psi\right. \\
& \left.+M \bar{\Psi} \Psi+i g_{A} \bar{\Psi} \gamma_{\mu} \gamma_{5} a_{\mu} \Psi\right\},
\end{aligned}
$$

where $M$ is the nucleon mass generated by spontaneous chiral symmetry breaking and $g_{A}$ is the coupling to the isovector axial current not constrained by chiral symmetry. As advertized, the nucleon mass term does not break chiral symmetry explicitly. This feature of the nonlinear realization will allow us to perform lattice investigations with massive fermions in the chiral limit.

\subsection{Lattice Formulation}

The contiuum discussion shows that the nonlinear realization of chiral symmetry does not rely on the anticommutation of the Dirac operator with $\gamma_{5}$. Therefore, the Nielsen-Ninomiya theorem does not apply and we can use a Wilson term to remove the fermion doublers without breaking chiral symmetry explicitly. The effective lattice action of the low-energy theory for nucleons and pions can thus be written in the following form:

$$
\begin{aligned}
& S[U, \bar{\Psi}, \Psi]=-\frac{F_{\pi}^{2}}{4} \sum_{x, \mu} \operatorname{Tr}\left[U_{x}^{\dagger} U_{x+\hat{\mu}}+U_{x+\hat{\mu}}^{\dagger} U_{x}\right] \\
& +\frac{1}{2} \sum_{x, \mu}\left(\bar{\Psi}_{x} \gamma_{\mu} V_{x, \mu} \Psi_{x+\hat{\mu}}-\bar{\Psi}_{x+\hat{\mu}} \gamma_{\mu} V_{x, \mu}^{\dagger} \Psi_{x}\right) \\
& +\frac{i g_{A}}{2} \sum_{x, \mu}\left(\bar{\Psi}_{x} \gamma_{\mu} \gamma_{5} A_{x, \mu}^{L} \Psi_{x}+\bar{\Psi}_{x+\hat{\mu}} \gamma_{\mu} \gamma_{5} A_{x, \mu}^{R} \Psi_{x+\hat{\mu}}\right) \\
& +\frac{r}{2} \sum_{x, \mu}\left(2 \bar{\Psi}_{x} \Psi_{x}-\bar{\Psi}_{x} V_{x, \mu} \Psi_{x+\hat{\mu}}-\bar{\Psi}_{x+\hat{\mu}} V_{x, \mu}^{\dagger} \Psi_{x}\right) \\
& +M \sum_{x} \bar{\Psi}_{x} \Psi_{x}
\end{aligned}
$$

with the Wilson term weighted by $r$. The lattice Goldstone boson field $U_{x}$, its square root $u_{x}$, and the nucleon field $\Psi_{x}$ and $\bar{\Psi}_{x}$ are obtainedtogether with the corresponding transformation behavior - by naive discretization. The continuum flavor "gauge" vector field $v_{\mu}(x)$ is realized by the flavor "gauge" parallel transporter

$$
\begin{aligned}
& V_{x, \mu}=\left(\tilde{V}_{x, \mu} \tilde{V}_{x, \mu}^{\dagger}\right)^{-1 / 2} \tilde{V}_{x, \mu}, \\
& \tilde{V}_{x, \mu}=\frac{1}{2}\left[u_{x}^{\dagger} u_{x+\hat{\mu}}+u_{x} u_{x+\hat{\mu}}^{\dagger}\right] .
\end{aligned}
$$

which is an element of the $S U\left(N_{f}=2\right)$ group even at finite lattice spacing and transforms under lattice flavor "gauge" transformations $V_{x}$ as

$V_{x, \mu} \rightarrow V_{x, \mu}^{\prime}=V_{x} V_{x, \mu} V_{x+\hat{\mu}}^{\dagger}$

The continuum axial vector field $a_{\mu}(x)$ is realized by the lattice fields $A_{x, \mu}^{L}$ and $A_{x, \mu}^{R}$ given in [2].

\section{APPLICATIONS}

The nonlinear realization of chiral symmetry on the lattice - illustrated above only for the simplest case - allows a non-perturbative description of flavor dynamics based on effective Lagrangians. This opens up a new class of investigations. 


\subsection{Low-Energy Effective Theories}

Within low-energy effective theories, we can study non-perturbative phenomena on the lattice such as the spectrum of the $S U\left(N_{f}\right)$ chiral rotor. While this can already be computed analytically in the continuum [7], lattice techniques will become crucial in more complicated effective theories describing, for example, nuclear matter.

\subsection{Static Baryons at Non-Zero $\mu$}

Beyond the strict validity range of low-energy effective theories, the presented methods can lead to new insights into the phase diagram of strongly interacting matter at non-vanishing chemical potential. In the limit of static baryons, interesting simplifications arise which we illustrate for $N_{f}=2$ in the continuum formulation.

A static nucleon, $M \rightarrow \infty$, at an undetermined position $\vec{x}$ (with $g_{A}=0$ ) is described by the spatial integral of the Polyakov loop,

$\Phi[U]=\int d^{3} x \operatorname{Tr}_{F} \mathcal{P} \exp \left[\int_{0}^{\beta} d t v_{4}(\vec{x}, t)\right]$,

where $\mathcal{P}$ denotes path ordering, $\beta=1 / T$ is the inverse temperature, and $F$ refers to the trace in the fundamental representation, which is realvalued for $S U\left(N_{f}=2\right)$. Then, taking also the limit of an infinite chemical potential $\mu \rightarrow \infty$ such that the difference $M-\mu$ remains finite, the grand canonical partition function for a system of pions in the background of static nucleons reduces to

$Z(\mu)=\int \mathcal{D} U \exp \left\{-S[U]+e^{-\beta(M-\mu)} \Phi[U]\right\}$.

As $\Phi[U]$ takes on real values only, the phase diagram of this model can be studied on the lattice without a complex action problem. Taking into account non-zero quark masses and the associated small explicit chiral symmetry breaking, the dependence of the phase structure on the quark masses can be determined as well. Since the static nucleon model shares all global symmetries with QCD, such investigations might help to extract the universal properties of the QCD phase diagram. To investigate the dependence on the strange quark mass, the model can be generalized to $S U\left(N_{f}=3\right)$. Again no complex action problem arises since now the baryons transform in the adjoint representation.

\subsection{Constituent Quarks on the Lattice}

A nonlinear realization of chiral symmetry was used also in the chiral quark model of Georgi and Manohar [4]. This model is obtained from (12) by replacing the nucleons with colored constituent quarks and by adding gluon dynamics. If this model provides an appropriate low-energy description for QCD, one could understand why the non-relativistic quark model works. Our lattice formulation [2] provides the ideal means to clarify this issue as there are open non-perturbative questions concerning, for example, the confinement of constituent quarks and the assumption of the chiral symmtry breaking scale $\Lambda_{\chi S B}$ being larger than the confinement scale $\Lambda_{\mathrm{QCD}}$.

\section{OUTLOOK AND CONCLUSION}

Is it interesting to ask if nonlinearly realized chiral symmetry can lead to a new approach to lattice QCD beyond low-energy effective theories. In order to answer this question, one may attempt to relate lattice QCD with GinspargWilson fermions to a lattice theory with explicit pion fields and a nonlineary realized chiral symmetry (cf. also [8]). In any case, the nonlinearly realized chiral symmetry provides an alternative perspective at chiral symmetry on the lattice.

\section{REFERENCES}

1. F. Niedermayer, Nucl. Phys. B (Proc. Suppl.) 73 (1999) 105 and references therein.

2. S. Chandrasekharan, M. Pepe, F. D. Steffen, and U.-J. Wiese, arXiv:hep-lat/0306020.

3. S. Weinberg, Phys. Rev. Lett. 18 (1967) 188; S. R. Coleman, J. Wess, and B. Zumino, Phys. Rev. 177 (1969) 2239; C. G. Callan, S. R. Coleman, J. Wess, and B. Zumino, ibid. 2247.

4. A. Manohar and H. Georgi, Nucl. Phys. B 234 (1984) 189.

5. S. Weinberg, Physica 96A (1979) 327.

6. J. Gasser and H. Leutwyler, Annals Phys. 158 (1984) 142; Nucl. Phys. B 250 (1985) 465.

7. H. Leutwyler, Phys. Lett. B 189 (1987) 197.

8. R. C. Brower, talk given at Lattice 2003. 